Теорія Ймовір. та Матем. Статист. Вип. 79, 2008
Theor. Probability and Math. Statist.

No. 79, 2009, Pages 107-116 S 0094-9000(09)00773-X

Article electronically published on December 28, 2009

\title{
APPROXIMATION OF FRACTIONAL BROWNIAN MOTION BY WIENER INTEGRALS
}

UDC 519.21

\author{
YU. S. MISHURA AND O. L. BANNA
}

\begin{abstract}
We find an approximation in the space $L_{\infty}\left([0, T] ; L_{2}(\Omega)\right)$ of a fractional Brownian motion by martingales of the form $\int_{0}^{t} a(s) d W_{s}$, where $W$ is a Wiener process, $a(s)$ is a power function with a negative index, that is $a(s)=k \cdot s^{-\alpha}$ where $k>0, \alpha=H-1 / 2$, and $H$ is the index of fractional Brownian motion.
\end{abstract}

\section{INTRODUCTION}

A Gaussian process $\left\{B_{t}^{H}, t \geq 0\right\}$ such that $B_{0}^{H}=0$ is called a fractional Brownian motion with the Hurst index $H \in(0,1)$ if $\mathrm{E} B_{t}^{H}=0$ and

$$
\mathrm{E} B_{t}^{H} B_{s}^{H}=\frac{1}{2}\left(t^{2 H}+s^{2 H}-|t-s|^{2 H}\right) .
$$

We consider the case of $H \in\left(\frac{1}{2}, 1\right)$. It is known that the fractional Brownian motion with the Hurst index $H \in\left(\frac{1}{2}, 1\right)$ is not a semimartingale. In particular, it is neither a martingale nor a process of bounded variation. A natural question arises on whether the fractional Brownian motion can be approximated in some metrics by martingales, semimartingales, or processes of bounded variation? The answer to this question is positive concerning an approximation by processes of bounded variation and semimartingales (the corresponding results are presented in the papers [1], [3], and [5]).

The best approximation in the space $L_{\infty}\left([0, T] ; L_{2}(\Omega)\right)$ of a fractional Brownian motion by martingales of the form $\int_{0}^{t} a(s) d W_{s}$ is found in the current paper, where $W$ is a Wiener process and $a(s)$ is a power function with a negative index, that is, a function of the form $a(s)=k \cdot s^{-\alpha}, k>0$.

More precisely, we consider the following problem. Let $T>0$ be a fixed number and consider the interval $[0, T]$. It is known [4 that the fractional Brownian motion $\left\{B_{t}^{H}, t \in[0, T]\right\}$ admits the representation

$$
B_{t}^{H}=\int_{0}^{t} z(t, s) d W_{s}
$$

where $\left\{W_{t}, t \in[0, T]\right\}$ is a Wiener process and

$$
z(t, s)=\left(H-\frac{1}{2}\right) c_{H} s^{1 / 2-H} \int_{s}^{t} u^{H-1 / 2}(u-s)^{H-3 / 2} d u .
$$

2000 Mathematics Subject Classification. Primary 60G15; Secondary 60G44.

Key words and phrases. Wiener integral, fractional Brownian motion. 
Here

$$
c_{H}=\left(\frac{2 H \cdot \Gamma\left(\frac{3}{2}-H\right)}{\Gamma\left(H+\frac{1}{2}\right) \Gamma(2-2 H)}\right)^{1 / 2}
$$

and $\Gamma(x), x>0$, denotes the gamma function. In what follows let $\alpha=H-\frac{1}{2}$.

Now let $a:[0, T] \rightarrow \mathbf{R}$ be a nonrandom measurable function of the class $L_{2}[0, T]$; that is, $a$ is such that the stochastic integral $\int_{0}^{t} a(s) d W_{s}, t \in[0, T]$, is well defined with respect to the same Wiener process $\left\{W_{t}, t \in[0, T]\right\}$ (this integral is usually called the Wiener integral if the integrand is a nonrandom function). The problem is to find

$$
\inf _{a \in L_{2}[0, T]} \sup _{0 \leq t \leq T} \mathrm{E}\left(B_{t}^{H}-\int_{0}^{t} a(s) d W_{s}\right)^{2} .
$$

In Section 2, we discuss the classes where one can try to find functions for the best approximation. In Section 3, we solve a simplified version of the above problem where the infimum is considered over all power functions $a(s)=k \cdot s^{-\alpha}, k>0$, instead of the whole class $L_{2}[0, T]$. We find the function where the infimum attains its value as well as the value of the infimum itself. Note that this value is nonzero.

\section{A COMPARISON OF SOME CLASSES OF FUNCTIONS FROM THE POINT OF VIEW OF THE BEST APPROXIMATION OF A FRACTIONAL BROWNIAN MOTION}

Let $\left\{B_{t}^{H}, t \geq 0\right\}$ be a fractional Brownian motion with the Hurst index $H \in\left(\frac{1}{2}, 1\right)$, a number $T>0$ be fixed, $a:[0, T] \rightarrow \mathbf{R}$ be a measurable function such that $a \in L_{2}[0, T]$, a square integrable martingale $\left\{M_{t}, t \in[0, T]\right\}$ be of the form $M_{t}=\int_{0}^{t} a(s) d W_{s}$, where $\left\{W_{t}, t \in[0, T]\right\}$ is the Wiener process related to fractional Brownian motion as follows: for all $t \in[0, T]$ we have

$$
B_{t}^{H}=\int_{0}^{t} z(t, s) d W_{s}
$$

where the kernel $z$ is such that

$$
z(t, s)=c_{H}(H-1 / 2) s^{1 / 2-H} \int_{s}^{t} u^{H-1 / 2}(u-s)^{H-3 / 2} d u .
$$

The existence of representation (1) is established in the paper 44. Here

$$
c_{H}=\left(\frac{2 H \cdot \Gamma\left(\frac{3}{2}-H\right)}{\Gamma\left(H+\frac{1}{2}\right) \Gamma(2-2 H)}\right)^{1 / 2} .
$$

We evaluate

$$
\min _{a \in \mathcal{K}} \max _{0 \leq t \leq T} \mathrm{E}\left(B_{t}^{H}-M_{t}\right)^{2},
$$

where $\mathcal{K} \subset L_{2}[0, T]$ is some class of functions.

Remark 2.1. The exact value of the $\min _{a \in L_{2}[0, T]} \max _{0 \leq t \leq T} \mathrm{E}\left(B_{t}^{H}-M_{t}\right)^{2}$ is still unknown.

Remark 2.2. In view of representation (1), it is natural to consider the integrals with respect to the same Wiener process $W$, since the distance $\mathrm{E}\left(B_{t}^{H}-M_{t}\right)^{2}$ increases if $M_{t}$ is of the form $M_{t}=\int_{0}^{t} a(s) d \widetilde{W}_{s}$, where the Wiener process $\widetilde{W}$ contains a component that is independent of $W$.

First we prove two auxiliary results concerning the functions $a$ in (2).

Lemma 2.1. If the Lebesgue measure of the set $A=\{t \in[0, T]: a(t)<0\}$ is positive, then the minimum in (2) is not attained at a function $a \in A$. 
Proof. We rewrite $\mathrm{E}\left(B_{t}^{H}-M_{t}\right)^{2}$ as follows:

$$
\mathrm{E}\left(B_{t}^{H}-M_{t}\right)^{2}=t^{2 H}-2 \int_{0}^{t} z(t, s) a(s) d s+\int_{0}^{t} a^{2}(s) d s .
$$

This makes it clear that if one changes $a(s)$ for $-a(s)$ at the points $s$ where $a(s)<0$, then the right hand side of (2) does not increase.

Lemma 2.2. Among all functions $a \in L_{2}[0, T]$ such that $a(s) \cdot s^{1 / 2-H}$ is nondecreasing, the minimum in (2) is attained at the function $a(s)=c_{H} s^{H-1 / 2}$.

Proof. We rewrite the right hand side of (3) as follows:

$$
\varphi(t):=t^{2 H}-2 c_{H} \alpha \int_{0}^{t} u^{H-1 / 2} \int_{0}^{u} s^{1 / 2-H}(u-s)^{H-3 / 2} a(s) d s d u+\int_{0}^{t} a^{2}(s) d s .
$$

Differentiating the right hand side of (4) with respect to $t$ we get

$$
\varphi^{\prime}(t)=2 H t^{2 H-1}-2 c_{H} \alpha t^{H-1 / 2} \int_{0}^{t} s^{1 / 2-H}(t-s)^{H-3 / 2} a(s) d s+a^{2}(t) .
$$

Changing the variable $s=t \cdot u$ in the latter integral, we obtain

$$
\varphi^{\prime}(t)=2 H t^{2 H-1}-2 c_{H} \alpha t^{H-1 / 2} \int_{0}^{1} s^{1 / 2-H}(1-s)^{H-3 / 2} a(t s) d s+a^{2}(t) .
$$

Let $s^{1 / 2-H} \cdot a(s)=: b(s)$. Then $a(s)=b(s) \cdot s^{H-1 / 2}$ and $\varphi^{\prime}(t)$ becomes of the form

$$
\varphi^{\prime}(t)=\psi(t) \cdot t^{2 H-1},
$$

where $\psi(t)=2 H-2 c_{H} \alpha \int_{0}^{1}(1-s)^{H-3 / 2} b(t s) d s+b^{2}(t)$.

If the function $b$ is nondecreasing with respect to $t$, then $b(t s) \leq b(t)$ for $t>0$ and $s \leq 1$. Thus

or

$$
\psi(t) \geq 2 H-2 c_{H} \alpha \int_{0}^{1}(1-s)^{H-3 / 2} d s \cdot b(t)+b^{2}(t)
$$

$$
\psi(t) \geq 2 H-2 c_{H} \cdot b(t)+b^{2}(t) .
$$

The bound $c_{H}^{2}<2 H$ is easy. Indeed, it follows from

$$
\Gamma\left(\frac{3}{2}-H\right)<\Gamma\left(H+\frac{1}{2}\right) \Gamma(2-2 H), \quad H \in\left(\frac{1}{2}, 1\right) .
$$

The latter inequality is obtained in the paper [2, Theorem 2]. Thus the discriminant $\frac{D}{4}=c_{H}^{2}-2 H$ of the quadratic polynomial $x^{2}-2 c_{H} x+2 H$ is negative, whence $\psi(t) \geq 0$ and the minimal value of $\psi(t)$ is attained at $b(t)=c_{H}$.

On the other hand, the bigger the derivative $\varphi^{\prime}(t)$ for all $t$, the bigger is $\varphi(t)$. Thus the minimum among all $a(s)$ such that $b(s)=a(s) s^{1 / 2-H}$ is nondecreasing is attained at $b(s)=c_{H}$. The lemma is proved.

Remark 2.3. Lemma 2.2 generalizes Theorem 2 of the paper 2 . It is proved in 2 that the minimum in (2) among all power functions $a(s)=k s^{H-1 / 2}$ is attained for $k=c_{H}$. Moreover, it is also proved in 2 that the minimum in (2) among all power functions with a negative index is attained at the constant function $a(t)=c_{1}(H) \cdot T^{\alpha}$, where $c_{1}(H)=\alpha c_{H} \cdot(\alpha+1)^{-1} \cdot B(1-\alpha, \alpha)$. Therefore it is worthwhile to consider the power functions with a negative index. 
3. An ApProximation of A FRACTIONAL BROWNiAn MOTION BY INTEGRALS OF POWER FUNCTIONS WITH A NEGATIVE INDEX

First we prove some auxiliary results.

Let

$$
f(t, k):=\mathrm{E}\left(B_{t}^{H}-k \int_{0}^{t} s^{-\alpha} d W_{s}\right)^{2} .
$$

Lemma 3.1. 1) The function $f(t, k)$ admits the following representation:

$$
f(t, k)=t^{2 H}-4 t k \frac{H}{c_{H}}+k^{2} \frac{t^{2-2 H}}{2-2 H} .
$$

2) For all $k>0$, the function $f(t, k)$ has a point of local maximum in $\mathbf{R}^{+}$, namely

$$
t_{1}=t_{1}(k)=k^{1 /(2 \alpha)}(p(H))^{-1 /(2 \alpha)},
$$

where

$$
p(H)=\frac{2 H}{c_{H}}+\sqrt{\left(\frac{2 H}{c_{H}}\right)^{2}-2 H}
$$

as well as the point of local minimum in $\mathbf{R}^{+}$, namely

$$
t_{2}=t_{2}(k)=k^{1 /(2 \alpha)}\left(p_{1}(H)\right)^{-1 /(2 \alpha)},
$$

where

$$
p_{1}(H)=\frac{2 H}{c_{H}}-\sqrt{\left(\frac{2 H}{c_{H}}\right)^{2}-2 H} .
$$

3) If $k_{\lim }:=T^{2 \alpha} p(H)$, then $t_{1}(k)<T$ for $k<k_{\lim } ; t_{1}(k)=T$ for $k=k_{\lim }$; and $t_{1}(k)>T$ for $k>k_{\text {lim }}$.

Remark 3.1. 1. We conclude from (6) and (7) that $t_{2}(k)>t_{1}(k)$.

2. The bound $\Gamma(1-\alpha)<\Gamma(\alpha+1) \Gamma(1-2 \alpha)$ is proved in [2, Theorem 2] for $\alpha=H-\frac{1}{2}$ and $\frac{1}{2}<H<1$. This bound implies, in particular, that

$$
\left(\frac{2 H}{c_{H}}\right)^{2}-2 H=\frac{2 H \Gamma(\alpha+1) \Gamma(1-2 \alpha)}{\Gamma(1-\alpha)}-2 H>0 .
$$

3. Relation (5) and Lemma 2.1 imply that the best approximation can be attained only for $k>0$. Thus the case of $k<0$ is not considered in the rest of the current paper.

4. It is obvious that $f(t, k)>0$ for all $t$ and $k$. In particular, we obtain for $t=1$ that the quadratic polynomial

$$
1-4 k \frac{H}{c_{H}}+\frac{k^{2}}{2-2 H}
$$

is positive. Its discriminant obviously is negative and equals

$$
D=\frac{16 H^{2}}{c_{H}^{2}}-\frac{2}{1-H} .
$$

This implies an interesting inequality, which will be used in what follows; namely,

$$
c_{H}^{2}>8 H^{2}(1-H)
$$

or

$$
\frac{\Gamma\left(\frac{3}{2}-H\right)}{\Gamma\left(H+\frac{1}{2}\right) \Gamma(2-2 H)}>4 H(1-H)
$$

or

$$
2 H<\frac{\Gamma\left(\frac{3}{2}-H\right)}{\Gamma\left(H+\frac{1}{2}\right) \Gamma(3-2 H)}, \quad H \in\left(\frac{1}{2}, 1\right)
$$


Moreover, the inequality becomes an equality at the points $H=\frac{1}{2}$ and $H=1$. Using

$$
\Gamma(2 a)=\Gamma(a) \Gamma\left(a+\frac{1}{2}\right) \frac{2^{2 a-1 / 2}}{(2 \pi)^{1 / 2}}
$$

we rewrite (8) in the following form:

$$
\frac{8 H}{\sqrt{\pi}}<\frac{4^{H}}{\Gamma\left(H+\frac{1}{2}\right) \Gamma(2-H)} .
$$

For example, the left hand side of (10) for $H=3 / 4$ equals $\frac{3}{2}$, while its right hand side is equal to

$$
\frac{\Gamma(3 / 4)}{\Gamma(5 / 4) \Gamma(3 / 2)}=\frac{8}{3 \sqrt{\pi}} \approx 1.5045
$$

Proof. Assertion 1) follows after straightforward calculations. We have

$$
\mathrm{E}\left(B_{t}^{H}-k \int_{0}^{t} s^{-\alpha} d W_{s}\right)^{2}=t^{2 H}-2 k \mathrm{E} B_{t}^{H} \int_{0}^{t} s^{-\alpha} d W_{s}+k^{2} \frac{t^{2-2 H}}{2-2 H}
$$

According to representation (11), $B_{t}^{H}=\int_{0}^{t} z(t, s) d W_{s}$. Then it is easy to check that

$$
\mathrm{E} B_{t}^{H} \int_{0}^{t} s^{-\alpha} d W_{s}=\int_{0}^{t} z(t, s) s^{-\alpha} d s=4 \frac{H}{c_{H}} t .
$$

To prove assertion 2) we differentiate $f(t, k)$ with respect to $t$ :

$$
\frac{\partial f(t, k)}{\partial t}=t^{2 \alpha}\left(2 H-t^{-2 \alpha} k \cdot \frac{4 H}{c_{H}}+k^{2} t^{-4 \alpha}\right)
$$

and make the change $x:=k t^{-2 \alpha}$. Then we obtain the quadratic equation

$$
x^{2}-\frac{4 H}{c_{H}} x+2 H=0
$$

whose roots are $x_{1}=p(H)$ and $x_{2}=p_{1}(H)$. This implies equalities (6) and (7). Since the derivative $\partial f / \partial t$ is positive for $t<t_{1}(k)$ and negative for $t_{1}(k)<t<t_{2}(k)$, we prove that $t_{1}(k)$ and $t_{2}(k)$ are the points of local maximum and minimum, respectively.

To prove assertion 3) we first find $k_{\text {lim }}$, that is, a point such that $t_{1}(k)=T$. It is obvious that $k_{\lim }=T^{2 \alpha} p(H)$. Since $t_{1}(k)$ increases with respect to $k$, we have $t_{1}(k)<T$ for $k<k_{\text {lim }}$, while $t_{1}(k)>T$ for $k>k_{\text {lim }}$. The lemma is proved.

Corollary 3.1. We have

$$
\max _{t \in[0, T]} f(t, k)= \begin{cases}f(T, k) & \text { if } t_{1}(k) \geq T, \\ \max \left\{f\left(t_{1}(k), k\right), f(T, k)\right\} & \text { if } t_{1}(k)<T .\end{cases}
$$

Consider some properties of the point $k_{\lim }$. We find $k_{\min }$, that is, a number $k$ such that $f(T, k)$ attains the minimum at $k_{\min }$ with respect to $k$ if $T$ is fixed. It is clear that

$$
k_{\min }=\frac{4 H(1-H)}{c_{H}} T^{2 \alpha} .
$$

Lemma 3.2. We have $k_{\min }<k_{\lim }$.

Proof. It is sufficient to prove that

$$
\frac{4 H(1-H)}{c_{H}}<p(H)
$$


Or

$$
\frac{2 H(1-2 H)}{c_{H}}<\sqrt{\left(\frac{2 H}{c_{H}}\right)^{2}-2 H .}
$$

The latter inequality obviously holds, since its left hand side is negative.

According to Corollary 3.1 and assertion 3) of Lemma 3.1, we find that

$$
\inf _{k>0} \max _{t \in[0, T]} f(t, k)=\min \left\{\inf _{0<k \leq k_{\lim }}\left\{f\left(t_{1}(k), k\right), f(T, k)\right\}, \inf _{k>k_{\lim }} f(T, k)\right\} .
$$

Thus we compare the graphs of the functions

$$
\varphi_{1}(k):=f\left(t_{1}(k), k\right) \text { and } \varphi_{2}(k):=f(T, k)
$$

for $0<k<k_{\text {lim. }}$.

First we prove an interesting property of these functions at the point $k=k_{\text {lim. }}$. We have $\varphi_{1}\left(k_{\lim }\right)=\varphi_{2}\left(k_{\lim }\right)$ by the definition of $k_{\lim }$. It turns out that the derivatives of these functions are the same at the point $k_{\mathrm{lim}}$.

Lemma 3.3. We have $\varphi_{1}^{\prime}\left(k_{\lim }\right)=\varphi_{2}^{\prime}\left(k_{\lim }\right)$.

Proof. It is clear that

$$
\begin{aligned}
\varphi_{1}(k) & =\left(t_{1}(k)\right)^{2 H}-\frac{4 k t_{1}(k) H}{c_{H}}+k^{2} \frac{\left(t_{1}(k)\right)^{2-2 H}}{2-2 H} \\
& =\left(k p(H)^{-1}\right)^{\frac{2 H}{2 H-1}}-\frac{4 k \cdot\left(k p(H)^{-1}\right)^{\frac{1}{2 H-1}} \cdot H}{c_{H}}+k^{2} \frac{\left(k p(H)^{-1}\right)^{\frac{2-2 H}{2 H-1}}}{2-2 H} \\
& =k^{\frac{2 H}{2 H-1}} \cdot c_{1}(H),
\end{aligned}
$$

where

$$
\begin{aligned}
c_{1}(H) & =(p(H))^{-\frac{2 H}{2 H-1}}\left(1-\frac{4 H p(H)}{c_{H}}+\frac{p^{2}(H)}{2-2 H}\right) \\
& =(p(H))^{-\frac{2 H}{2 H-1}} \cdot \frac{2 H-1}{1-H} \cdot\left(\frac{2 H}{c_{H}} p(H)-1\right) .
\end{aligned}
$$

Further,

$$
\varphi_{2}(k)=T^{2 H}-\frac{4 k H}{c_{H}} T+k^{2} \frac{T^{2-2 H}}{2-2 H}
$$

Hence

$$
\varphi_{1}^{\prime}(k)=\frac{2 H}{2 H-1} k^{\frac{1}{2 H-1}} c_{1}(H)
$$

and

$$
\varphi_{2}^{\prime}(k)=\frac{k T^{2-2 H}}{1-H}-\frac{4 H}{c_{H}} T .
$$

Substituting $k_{\lim }$ in place of $k$, we get

$$
\begin{aligned}
\varphi_{1}^{\prime}\left(k_{\lim }\right) & =\frac{2 H}{2 H-1} T p(H)^{\frac{1}{2 H-1}} \cdot p(H)^{-\frac{2 H}{2 H-1}}\left(1-\frac{4 H p(H)}{c_{H}}+\frac{p^{2}(H)}{2-2 H}\right) \\
& =\frac{2 H}{2 H-1} T\left(p(H)^{-1}-\frac{4 H}{c_{H}}+\frac{p(H)}{2-2 H}\right) .
\end{aligned}
$$

Similarly

$$
\varphi_{2}^{\prime}\left(k_{\lim }\right)=T\left(\frac{p(H)}{1-H}-\frac{4 H}{c_{H}}\right) .
$$


It remains to note that $p(H)$ is a root of equation (1), that is,

$$
p^{2}(H)=\frac{4 H}{c_{H}} p(H)-2 H
$$

Then we rewrite $\varphi_{1}^{\prime}\left(k_{\lim }\right)$ as follows:

$$
\varphi_{1}^{\prime}\left(k_{\lim }\right)=\frac{2 H}{1-H}\left(\frac{2 H}{c_{H}}-p(H)^{-1}\right) T .
$$

Finally

$\frac{p(H)}{1-H}-\frac{4 H}{c_{H}}-\frac{4 H^{2}}{c_{H}(1-H)}+\frac{2 H}{1-H} p(H)^{-1}=\frac{p(H)^{-1}}{1-H}\left(p^{2}(H)-\frac{4 H}{c_{H}} p(H)+2 H\right)=0$, and this means that the derivatives are equal. The lemma is proved.

Now we show that $k_{\text {lim }}$ is the largest point of intersection of the functions $\varphi_{1}(k)$ and $\varphi_{2}(k)$. This follows from the following result applied together with

$$
\varphi_{1}\left(k_{\lim }\right)=\varphi_{2}\left(k_{\lim }\right) \quad \text { and } \quad \varphi_{1}^{\prime}\left(k_{\lim }\right)=\varphi_{2}^{\prime}\left(k_{\lim }\right) .
$$

Lemma 3.4. For all $k \geq k_{\lim }, \varphi_{1}^{\prime \prime}(k)>\varphi_{2}^{\prime \prime}(k)$.

Proof. Relations (12) and (13) imply that

$$
\varphi_{1}^{\prime \prime}(k)=\frac{2 H}{(2 H-1)^{2}} k^{\frac{2-2 H}{2 H-1}} c_{1}(H)
$$

and that $\varphi_{1}^{\prime \prime}(k)$ increases with respect to $k$, while $\varphi_{2}^{\prime \prime}(k)=\frac{T^{2-2 H}}{1-H}$. At the point $k=k_{\lim }$,

$$
\begin{aligned}
\varphi_{1}^{\prime \prime}\left(k_{\lim }\right) & =\frac{2 H}{(2 H-1)^{2}} T^{2-2 H}(p(H))^{\frac{2-2 H}{2 H-1}} c_{1}(H) \\
& =\frac{2 H}{(1-H)(2 H-1)} T^{2-2 H}(p(H))^{-2}\left(\frac{2 H}{c_{H}} p(H)-1\right) .
\end{aligned}
$$

It is sufficient to prove that

$$
\frac{2 H}{2 H-1}\left(\frac{2 H}{c_{H}} p(H)-1\right)>p^{2}(H)
$$

or

$$
\frac{2 H}{2 H-1}\left(\frac{2 H}{c_{H}} p(H)-1\right)>\frac{4 H}{c_{H}} p(H)-2 H .
$$

The latter bound is equivalent to the inequality $c_{H}^{2}<2 H$. This result is obtained in the paper [2, Theorem 2].

Now we show that there exists a unique point $0<k^{*}<k_{\text {lim }}$ of intersection of the functions $\varphi_{1}(k)$ and $\varphi_{2}(k)$. This means that $\varphi_{1}(k)<\varphi_{2}(k)$ for $0<k<k^{*}$ and

$$
\varphi_{1}(k)>\varphi_{2}(k) \text { for } k^{*}<k<k_{\lim }
$$

and that $k_{\lim }<k<\infty$.

Lemma 3.5. There exists a unique point $k^{*}$ such that $0<k^{*}<p_{1}(H) T^{2 \alpha}$ and

$$
\varphi_{1}\left(k^{*}\right)=\varphi_{2}\left(k^{*}\right) .
$$

Proof. The point $k^{*}$ necessarily satisfies the relations

$$
\left\{\begin{array}{l}
\left(k^{*}\right)^{\frac{2 H}{2 H-1}} c_{1}(H)-\left(k^{*}\right)^{2} \frac{T^{2-2 H}}{2-2 H}+4 k^{*} T \frac{H}{c_{H}}-T^{2 H}=0 \\
\frac{2 H}{2 H-1}\left(k^{*}\right)^{\frac{1}{2 H-1}} c_{1}(H)-k^{*} \frac{T^{2-2 H}}{1-H}+4 T \frac{H}{c_{H}}>0
\end{array}\right.
$$


whence, in particular, we obtain

$$
\frac{2 H}{2 H-1}\left(k^{*} \frac{T^{2-2 H}}{2-2 H}-4 T \frac{H}{c_{H}}+\frac{T^{2 H}}{k^{*}}\right)-k^{*} \frac{T^{2-2 H}}{1-H}+4 T \frac{H}{c_{H}}>0 .
$$

The latter inequality can be rewritten as follows:

$$
k^{*} T^{1-2 H}-4 \frac{H}{c_{H}}+2 H \frac{T^{2 H-1}}{k^{*}}>0 .
$$

Denoting $y:=k^{*} T^{1-2 H}$, we obtain that the inequality $y^{2}-\frac{4 H}{c_{H}} y+2 H>0$ is true for $y>p(H)$ or, equivalently, for $y<p_{1}(H)$. Note that if $y>p(H)$, then

$$
k^{*}>T^{2 \alpha} p(H)=k_{\lim }
$$

and the preceding lemmas imply that there are no points of intersection greater than $k_{\text {lim. }}$. It remains to consider the behavior of the function

$$
\theta(x):=x^{\frac{2 H}{2 H-1}} c_{1}(H)-x^{2} \frac{T^{2-2 H}}{2-2 H}+4 x T \frac{H}{c_{H}}-T^{2 H}
$$

in the interval $\left(0, p_{1}(H) T^{2 \alpha}\right)$. It is obvious that $\theta(0)<0$. Thus it is sufficient to show that $\theta\left(p_{1}(H) T^{2 \alpha}\right)>0$, whence the existence of the point $k^{*}$ follows (its existence is obvious).

Rewrite $\theta\left(p_{1}(H) T^{2 \alpha}\right)$ as follows:

$$
\begin{aligned}
& \theta\left(p_{1}(H) T^{2 \alpha}\right) \\
& \quad=T^{2 H}\left(\left(\frac{p_{1}(H)}{p(H)}\right)^{\frac{2 H}{2 H-1}} \frac{2 H-1}{1-H}\left(\frac{2 H}{c_{H}} p(H)-1\right)-\frac{\left(p_{1}(H)\right)^{2}}{2-2 H}+4 \frac{p_{1}(H) H}{c_{H}}-1\right) .
\end{aligned}
$$

For simplicity put $u:=p(H)>z:=p_{1}(H)$. We need to prove the inequality

$$
\left(\frac{z}{u}\right)^{\frac{2 H}{2 H-1}} \frac{2 H-1}{1-H}\left(\frac{2 H}{c_{H}} u-1\right)-\frac{z^{2}}{2-2 H}+4 \frac{z H}{c_{H}}-1>0,
$$

where $z^{2}=\frac{4 H}{c_{H}} z-2 H$. Thus inequality (14) is equivalent to

$$
z^{\frac{2 H}{2 H-1}}\left(\frac{2 H}{c_{H}} u-1\right)>u^{\frac{2 H}{2 H-1}}\left(\frac{2 H}{c_{H}} z-1\right)
$$

or

$$
\frac{4 H^{2}}{c_{H}} z^{\frac{1}{2 H-1}}-z^{\frac{2 H}{2 H-1}}>\frac{4 H^{2}}{c_{H}} u^{\frac{1}{2 H-1}}-u^{\frac{2 H}{2 H-1}},
$$

since $z u=2 H$. Consider the function

$$
\beta(s):=\frac{4 H^{2}}{c_{H}} s^{\frac{1}{2 H-1}}-s^{\frac{2 H}{2 H-1}} .
$$

Its derivative

$$
\beta^{\prime}(s)=\frac{1}{2 H-1} s^{\frac{2-2 H}{2 H-1}}\left(\frac{4 H^{2}}{c_{H}}-2 H s\right)
$$

is equal to zero at the point $s_{0}=\frac{2 H}{c_{H}}$ and $\beta(u)$ attains its maximum at this point. The points $u$ and $z$ are symmetric with respect to $s_{0}$. The absolute value of the derivative $\beta^{\prime}(s)$ at the point $s_{0}+\alpha$ is equal to

if $\alpha>0$, or to

$$
\frac{1}{2 H-1}\left(s_{0}+\alpha\right)^{\frac{2-2 H}{2 H-1}}|2 H \alpha|
$$

$$
|2 H \alpha| \frac{1}{2 H-1}\left(s_{0}-\alpha\right)^{\frac{2-2 H}{2 H-1}}<|2 H \alpha| \frac{1}{2 H-1}\left(s_{0}+\alpha\right)^{\frac{2-2 H}{2 H-1}}
$$




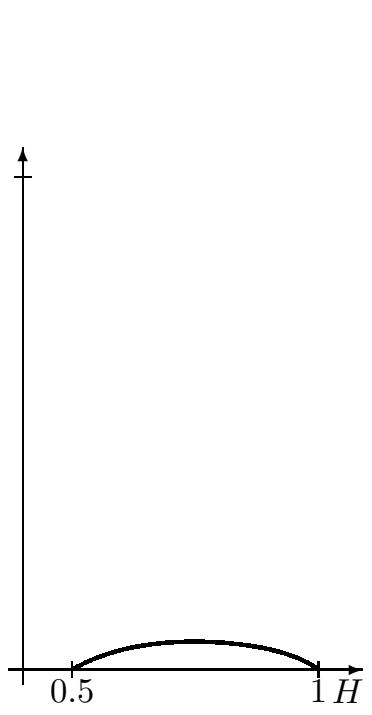

FiguRe 1

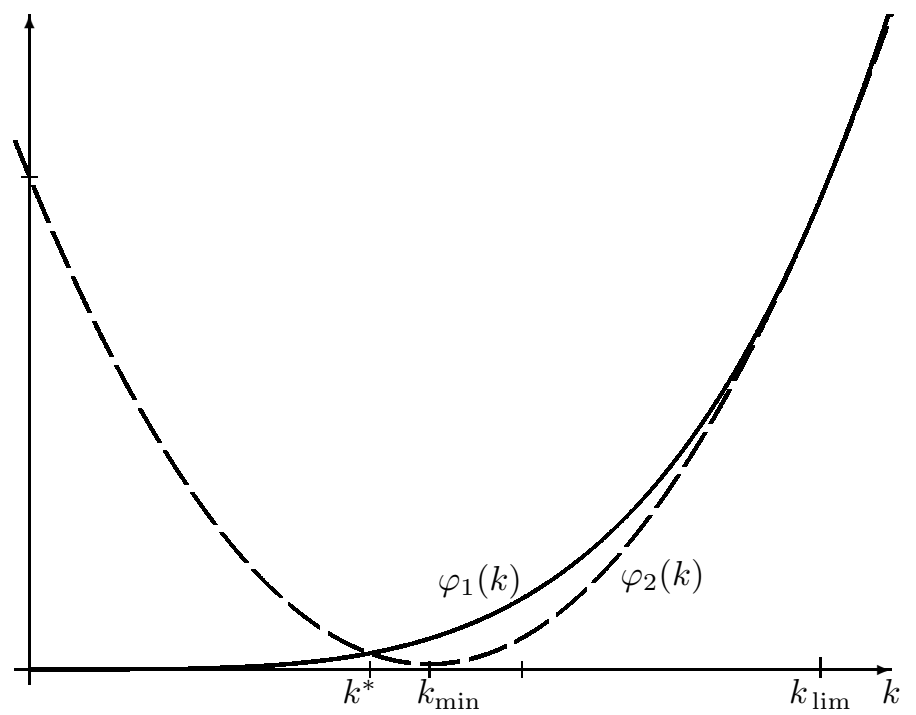

FIGURE 2

if $\alpha<0$. This implies that $\beta(z)>\beta(u)$, which is equivalent to inequality (15). The lemma is proved.

Remark 3.2. One can show that $k_{\min }<p_{1}(H) T^{2 \alpha}$. This bound is equivalent to the inequality

$$
\frac{4 H(1-H)}{c_{H}}<\frac{2 H}{c_{H}}-\sqrt{\left(\frac{2 H}{c_{H}}\right)^{2}-2 H}
$$

or

$$
\sqrt{\left(\frac{2 H}{c_{H}}\right)^{2}-2 H}<\frac{4 H^{2}-2 H}{c_{H}}
$$

or $-2 H<\left(16 H^{4}-16 H^{3}\right) /\left(c_{H}^{2}\right)$. The latter inequality is equivalent to $c_{H}^{2}>8 H^{2}(1-H)$, and this one was proved in Remark 3.1. Note that the inequality $k_{\min }<p_{1}(H) T^{2 \alpha}$ improves Lemma 3.2 essentially.

Remark 3.3. We are unable to provide an analytic proof of the inequality $k_{\min }>k^{*}$, which, in fact, is equivalent to $\varphi_{1}\left(k_{\min }\right)>\varphi_{2}\left(k_{\min }\right)$ or

$$
\left(k_{\min }\right)^{\frac{2 H}{2 H-1}} c_{1}(H)+\frac{8 H^{2}(1-H)}{c_{H}^{2}} T^{2 H}-T^{2 H}>0 .
$$

In Figure 3, we depict the graph of the function $\varphi_{1}\left(k_{\min }\right)-\varphi_{2}\left(k_{\min }\right)$. Its values are calculated up to the fourth decimal digit. The graph clearly shows that the desired inequality holds for $\frac{1}{2}<H<1$. Note that the latter inequality improves essentially inequality (14). According to this inequality, the graphs of the functions $\varphi_{1}(k)$ and $\varphi_{2}(k)$ are as shown in Figure 3 for $H=\frac{2}{3}$. For other $H$, the graphs are similar.

Now we prove the main result.

Theorem 3.1. Let $\mathcal{K}=\left\{a(s)=k s^{1 / 2-H}, k>0\right\}$. Then

$$
\min _{a \in \mathcal{K}} \max _{0 \leq t \leq T} \mathrm{E}\left(B_{t}^{H}-M_{t}\right)^{2}=\varphi_{1}\left(k^{*}\right)
$$


and the minimum is attained at the function $a(s)=k^{*} s^{1 / 2-H}$, where $k^{*}$ is the smallest of the two points of intersection of the functions $\varphi_{1}(k)=k^{\frac{2 H}{2 H-1}} c_{1}(H)$, where

$$
c_{1}(H)=(p(H))^{-\frac{2 H}{2 H-1}} \frac{2 H-1}{1-H}\left(\frac{2 H}{c_{H}} p(H)-1\right)
$$

and

$$
\varphi_{2}(k)=T^{2 H}-\frac{4 k H}{c_{H}} T+k^{2} \frac{T^{2-2 H}}{2-2 H} .
$$

Proof. We apply Corollary 3.1 and Remark 3.3. If $k \leq k_{\text {lim }}$, then

$$
\max \left\{f\left(t_{1}(k), k\right), f(T, k)\right\}=\max \left\{\varphi_{1}(k), \varphi_{2}(k)\right\}=\varphi_{2}(k) \mathbf{1}_{k<k^{*}}+\varphi_{1}(k) \mathbf{1}_{k>k^{*}} .
$$

If $k<k^{*}$, then Lemma 3.5 implies that $\varphi_{2}(k)>\varphi_{2}\left(k^{*}\right)$; otherwise, if $k>k^{*}$, then $\varphi_{1}(k)>\varphi_{1}\left(k^{*}\right)\left(\right.$ and $\left.\varphi_{1}\left(k^{*}\right)=\varphi_{2}\left(k^{*}\right)\right)$. Finally if $k>k_{\text {lim }}$, then

$$
\varphi_{2}(k)=f(T, k)>\varphi_{2}\left(k_{\lim }\right)=\varphi_{1}\left(k_{\lim }\right)>\varphi_{1}\left(k^{*}\right) .
$$

Therefore $\min _{k} \max _{t \in[0, T]} f(t, k)=\varphi_{1}\left(k^{*}\right)\left(=\varphi_{2}\left(k^{*}\right)\right)$. The theorem is proved.

\section{BIBLIOGRAPHY}

1. T. Androshchuk, Approximation of a stochastic integral with respect to fractional Brownian motion by integrals with respect to absolutely continuous processes, Teor. Imovir. Mat. Stat. 73 (2005), 11-20; English transl. in Theory Probab. Math. Statist. 73 (2006), 19-29. MR2213333 (2006m:60072)

2. O. L. Banna and Yu. S. Mishura, The simplest martingales for the best approximation to the fractional Brownian motion, Visnyk Kyiv Shevchenko Univ., ser. matem. mekh. (2008), 38-43. (Ukrainian)

3. T. Androshchuk and Y. S. Mishura, Mixed Brownian-fractional Brownian model: absence of arbitrage and related topics, Stochastics 78 (2006), 281-300. MR2270939 (2007k:60198)

4. I. Norros, E. Valkeila, and J. Virtamo, An elementary approach to a Girsanov formula and other analytical results on fractional Brownian motions, Bernoulli 5(4) (1999), 571-587. MR1704556 (2000f:60053)

5. T. H. Thao, A note on fractional Brownian motion, Vietnam J. Math. 31 (2003), no. 3, 255260. MR2010525 (2004j:60081)

Department of Probability Theory and Mathematical Statistics, Faculty for Mechanics and Mathematics, National Taras Shevchenko University, Academician Glushkov Avenue 6, KYIV 03127, UKRAINE

E-mail address: myus@univ.kiev.ua

Department of Probability Theory and Mathematical Statistics, Faculty for Mechanics and Mathematics, National Taras Shevchenko University, Academician Glushkov Avenue 6, KYIV 03127, UKRAINE

E-mail address: bannaya@mail.univ.kiev.ua

Received 17/SEP/2007

Translated by OLEG KLESOV 